\title{
Population-Based Study of Bloodstream Infection Incidence and Mortality Rates, Finland, 2004-2018
}

\author{
Keiju S.K. Kontula, Kirsi Skogberg, Jukka Ollgren, Asko Järvinen, Outi Lyytikäinen
}

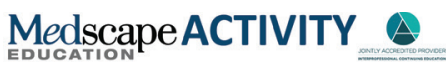

In support of improving patient care, this activity has been planned and implemented by Medscape, LLC and Emerging Infectious Diseases. Medscape, LLC is jointly accredited by the Accreditation Council for Continuing Medical Education (ACCME), the Accreditation Council for Pharmacy Education (ACPE), and the American Nurses Credentialing Center (ANCC), to provide continuing education for the healthcare team.

Medscape, LLC designates this Journal-based CME activity for a maximum of 1.00 AMA PRA Category 1 Credit(s) $^{\mathrm{TM}}$. Physicians should claim only the credit commensurate with the extent of their participation in the activity.

Successful completion of this CME activity, which includes participation in the evaluation component, enables the participant to earn up to $1.0 \mathrm{MOC}$ points in the American Board of Internal Medicine's (ABIM) Maintenance of Certification (MOC) program. Participants will earn MOC points equivalent to the amount of CME credits claimed for the activity. It is the CME activity provider's responsibility to submit participant completion information to ACCME for the purpose of granting ABIM MOC credit.

All other clinicians completing this activity will be issued a certificate of participation. To participate in this journal CME activity: (1) review the learning objectives and author disclosures; (2) study the education content; (3) take the post-test with a $75 \%$ minimum passing score and complete the evaluation at http://www.medscape.org/journal/eid; and (4) view/print certificate. For CME questions, see page $X X X$.

Release date: September 15, 2021; Expiration date: September 15, 2022

\section{Learning Objectives}

Upon completion of this activity, participants will be able to:

- Describe incidence and outcome of bloodstream infections (BSIs) in Finland during 2004 to 2018, according to an analysis of national, laboratory-based surveillance data

- Identify causative agents of BSIs in Finland during 2004-2018, according to an analysis of national, laboratory-based surveillance data

- Determine clinical and public health implications of incidence, outcome, causative agents, and trends of BSIs in Finland during 2004-2018, according to an analysis of national, laboratory-based surveillance data

\section{CME Editor}

Amy J. Guinn, BA, MA, Copyeditor, Emerging Infectious Diseases. Disclosure: Amy J. Guinn, BA, MA, has disclosed no relevant financial relationships.

\section{CME Author}

Laurie Barclay, MD, freelance writer and reviewer, Medscape, LLC. Disclosure: Laurie Barclay, MD, has disclosed no relevant financial relationships.

\section{Authors}

Disclosures: Keiju S.K. Kontula, MD; Jukka Ollgren, MS; and Outi Lyytikäinen, MD, PhD, have disclosed no relevant financial relationships. Kirsi Skogberg, MD, has disclosed the following relevant financial relationships: owns stock, stock options, or bonds from Nordea; Oriola; Orion Corporation. Asko Järvinen, MD, has disclosed the following relevant financial relationships: served as an advisor or consultant for Gilead Sciences, Inc.; GlaxoSmithKline; Sanofi; served as a speaker or member of a speakers bureau for: Astellas Pharma, Inc.; Gilead Sciences, Inc.

Author affiliations: Helsinki University Hospital, Helsinki, Finland (K.S.K. Kontula, K. Skogberg, A. Järvinen); Finnish Institute for Health and Welfare, Helsinki (J. Ollgren, O. Lyytikäinen) 
We evaluated the incidence, outcomes, and causative agents of bloodstream infections (BSI) in Finland during $2004-2018$ by using data from the national registries. We identified a total of $173,715 \mathrm{BSI}$; annual incidence increased from 150 to 309 cases $/ 100,000$ population. BSI incidence rose most sharply among persons $\geq 80$ years of age. The 1 -month case-fatality rate decreased from $13.0 \%$ to $12.6 \%$, but the 1-month all-cause mortality rate rose from 20 to 39 deaths $/ 100,000$ population. BSIs caused by Escherichia coli increased from $26 \%$ to $30 \%$ of all BSIs. BSIs caused by multidrug-resistant microbes rose from $0.4 \%$ to $2.8 \%$, mostly caused by extended-spectrum $\beta$-lactamase-producing $E$. coli. We observed an increase in community-acquired BSIs, from $67 \%$ to $78 \%$. The proportion of patients with severe underlying conditions rose from $14 \%$ to $23 \%$. Additional public health and healthcare prevention efforts are needed to curb the increasing trend in community-acquired BSIs and antimicrobial drug-resistant $E$. coli.

B loodstream infections (BSIs) are a major cause of illness and death worldwide. The incidence of BSIs has increased over time and reported BSI rates range from 122 to 220 cases/100,000 population (1-7). Rising incidence is probably related to an aging population and an increasing prevalence of underlying conditions and invasive procedures.

Despite advances in antimicrobial drug therapy, intensive care, and prevention strategies, BSIs cause an estimated 250,000 deaths annually in North America and Europe combined (8). Recent studies have reported BSI mortality rates of 21-32 deaths $/ 100,000$ population $(3,6)$ and 1-month casefatality rates of $\approx 17 \%-28 \%$ for nosocomial BSIs and $10 \%-19 \%$ for community-acquired BSIs (9-12). Higher mortality rates of $40 \%-50 \%$ have been observed in surveys of patients with BSIs or septic shock in hospital intensive care units $(13,14)$. However, only limited population-based data are available concerning the incidence and outcome of BSIs $(1,3,4,6,10,12,15)$. Most other reports mainly focus on selected hospitals or hospital units, a specific causative agent, or either healthcare-associated or community-acquired BSIs, and thus those studies represent select patient populations.

We used data from the national, laboratorybased surveillance system in Finland to analyze the annual incidence, causative agents, and outcomes of all BSIs in the country during 2004-2018. Our objective was to assess the burden and temporal trends of BSIs in Finland and identify targets for preventive interventions.

\section{Materials and Methods}

\section{Study Setting and Population}

Finland had a population 5.2 million in 2004 and 5.5 million in 2018. The country's healthcare system is organized into 20 healthcare districts; there are 5 tertiary care hospitals and 15 secondary care hospitals, and the number of primary care hospitals varies by district. All clinical microbiology laboratories in Finland report all bacterial and fungal isolates from blood samples to the National Infectious Disease Register (NIDR) (16). These notifications are sent electronically and comprise specimen date; type of microbe; and the patient's date of birth, sex, place of residence, and national identity code, a unique number given to each resident in Finland. NIDR merges multiple notifications of the same microbe from the same national identity code, indicating samples came from same person, and creates a single case if notifications occur within 3 months of each other.

In this retrospective study, we used NIDR data to identify all BSIs in Finland during 2004-2018. We included 187,553 BSIs with valid national identity codes in the study; we excluded 155 duplicate notifications, that is, those with same specimen date, microbe, and identity code (Figure 1). We retrieved date of death from the Population Information System (https:// dvv.fi/en/population-information-system) by linking the patient's national identity code with database information. We obtained information on patient hospitalization, including origin of the infection, and current and prior (1 year) diagnosis codes by linking to the National Hospital Discharge Register (HILMO).

The Ethics Committee of the Finnish Institute for Health and Welfare granted permission to analyze and link data from the NIDR and HILMO (approval no. THL/1349/6.02.00/2019). Because the data were already anonymized, patient informed consent was waived.

\section{Definitions}

We defined the presence of BSI as occurrence of viable bacteria or fungi in bloodstream evidenced by positive blood cultures. We defined polymicrobial BSI as isolation of $>1$ bacterial or fungal species in blood cultures collected within 2 days (16).

We classified BSI as healthcare-associated when the first blood culture was obtained $>2$ days after admission to hospital or within 2 days after discharge (17). We also classified cases as healthcare-associated for patients who came from another healthcare facility. We classified cases as community-acquired when patients had no prior hospitalization and 


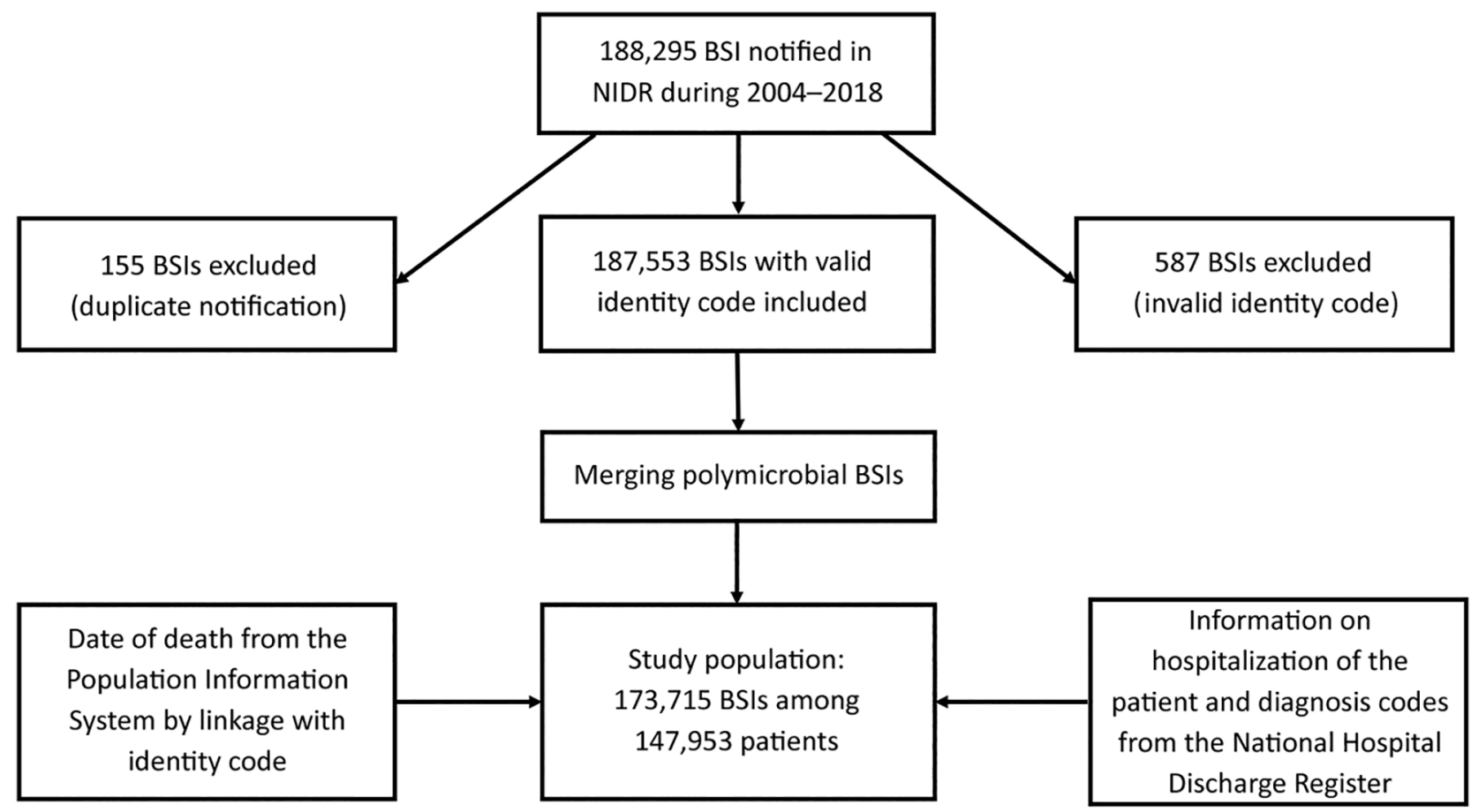

Figure 1. Flowchart of the data reviewed for inclusion in a study of the incidence of BSIs, Finland, 2004-2018. BSI, bloodstream infections; NIDR, National Infectious Disease Register.

blood culture specimens were collected $\leq 2$ days after hospital admission.

We defined underlying illness by using a validated algorithm for the Charlson comorbidity index (CCI) based on the International Classification of Diseases, 10th Revision $(18,19)$. We scored underlying illness on 3 levels: low (CCI score of 0 ) for patients with no reported underlying diseases, medium (CCI score 1-2), or high (CCI score $\geq 3$ ) (10). We defined all-cause mortality and case-fatality as death of a particular patient $\leq 30$ days after the first positive blood culture.

We defined the following bacteria as multidrug-resistant (MDR) microbes: extended-spectrum $\beta$-lactamase-producing (ESBL) Escherichia coli and Klebsiella pneumoniae, methicillin-resistant Staphylococcus aureus (MRSA), vancomycin-resistant Enterococcus (VRE) and carbapenemase-producing Enterobacteriaceae (CPE). We defined ESBL-E. coli and ESBL-K. pneumoniae as resistant or intermediately susceptible to third-generation cephalosporins. We defined CPE as E. coli, K. pneumoniae, and Enterobacter sp. resistant or intermediately susceptible to carbapenems.

\section{Analyses and Statistics}

We used population data from Statistics Finland (https://www.stat.fi/index_en.html) as denominators to calculate age- and sex-specific BSI incidence and mortality rates. We determined average annual incidence and mortality rates according to the total number of BSI episodes, BSI deaths, and population during 2004-2018. We applied a Poisson regression model, or negative binomial regression model in case of overdispersion, to compare the observed trends in annual incidence and mortality rates and used a log-linear binomial regression model for case-fatality proportions. We analyzed the data by using SPSS Statistics 25 (IBM, https://www.ibm.com) and Stata 16 (StataCorp LLC, https://www.stata.com).

\section{Results}

During 2004-2018, we identified a total of 173,715 BSIs among 147,953 patients in the NIDR (Figure 1 ). Median age among BSI patients was 70 (range $0-110$ ) years; $52 \%$ were male and $48 \%$ female. Among all BSIs, $7,568(4.4 \%)$ occurred in children $<16$ years of age, including 3,734 BSIs in infants $<1$ year of age. The average annual incidence was 216 BSI episodes/100,000 population and was higher among male (228 episodes $/ 100,000$ population) than female (203 episodes/100,000 population) patients. Overall BSI incidence was highest among patients $\geq 60$ years of age (618 cases $/ 100,000$ population) and patients $<1$ year of age (431 cases/100,000 population). Among infants $<1$ year and persons $\geq 40$ years of age, BSI 
incidence rates were higher in male than in female patients; only among persons 20-29 years of age was BSI incidence higher in female patients.

During 2004-2018, the annual BSI incidence rose from 150 to 309 cases/100,000 population, an average annual increase of $5.2 \%$ (95\% CI $4.8 \%$ $5.5 \% ; \mathrm{p}<0.01)$. BSI incidence increased in both sexes; among male persons, incidence increased from 155 to 327 cases $/ 100,000$ population, an average annual increase of $5.3 \%$ (95\% CI $4.9 \%-5.7 \%$; p $<0.01$ ); among female persons, incidence increased from 145 to 291 cases $/ 100,000$ population, an average annual increase of $5.0 \%$ (95\% CI $4.6 \%-5.4 \%$; p $<0.01)$ (Figure 2). The increase in the annual incidence was most prominent among persons $\geq 90$ years of age, from 1,155 to 3,005 cases $/ 100,000$ population, an average annual increase of $8.6 \%$ (95\% CI 8.0\%$9.1 \% ; \mathrm{p}<0.01)$. We observed a decreasing incidence only among infants $<1$ year of age, from 528 to 317 cases/100,000 population, an average annual decrease of $3.3 \%(p<0.01)$. We also noted a decreasing incidence in children $<10$ years of age, from 37 to 28 cases $/ 100,000$ population, an average annual decrease of $4.0 \%(\mathrm{p}<0.01)$.

Among all reported BSI cases, 22,474 (13\%) were fatal within 1 month; case-fatality rate was higher among male $(13.7 \%)$ than female $(12.1 \%)$ patients (relative risk 1.14, 95\% CI 1.11-1.17; $\mathrm{p}<0.01$ ). During 2004-2018, we noted a minor decrease in the 1-month case-fatality rate, from $13.0 \%$ to $12.6 \%$, an average annual relative reduction of $0.4 \%$ (95\% CI $0.1 \%-0.7 \%$; $\mathrm{p}<0.01$ ) (Figure 3). Among children and adolescents $1-19$ years of age and among persons $\geq 90$ years of age, the case-fatality rate increased slightly, but in other age groups we observed a descending rate. The average annual BSI mortality rate was 28 deaths/100,000

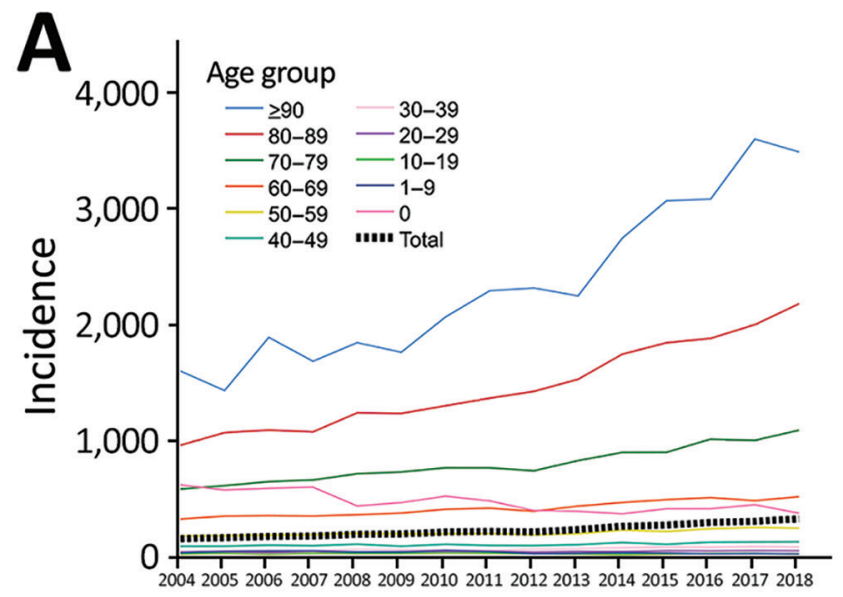

population during the study period. The mortality rate was higher for male patients in all age groups; among persons $\geq 20$ years of age, mortality rates were $>1.5$-fold higher among male than female patients. The mortality rate increased with age; among persons $\geq 70$ years of age, the rate was 148 deaths/100,000 population. The annual BSI mortality rate rose from 20 to 39 deaths/100,000 population during 2004-2018, and the overall average annual increase was 4.8\% (95\% CI $4.5 \%-5.1 \%$; $<<0.01$ ) (Figure 4 ); the increase was $4.5 \%(p<0.01)$ among male patients and $5.2 \%(p<0.01)$ among female patients. The increase in mortality rate was most notable among persons $\geq 90$ years of age, an average increase of $8.1 \%(\mathrm{p}<0.01)$.

Among all BSIs, gram-positive bacteria caused $46 \%$ of infections, gram-negative bacteria $46 \%$, fungi $1.5 \%$, and other unclassified bacteria $0.2 \%$. Polymicrobial BSIs accounted for $7 \%$ of all BSIs. E. coli was the most common causative pathogen $(29 \%$ of all BSIs), but other identified pathogens included S. aureus $(13 \%)$, coagulase-negative staphylococcus (CNS) $(8 \%)$, $\beta$-hemolytic streptococci $(8 \%)$, Streptococcus pneumoniae (7\%), Klebsiella sp. (5\%), and enterococci (4\%). Gram-positive bacteria were the most common cause of BSIs in male patients (52\% vs. $40 \%$ for female patients), whereas gram-negative bacteria were more prevalent in female patients (53\% vs. 39\% for male patients). Polymicrobial BSIs were more frequently noted in male patients than in female patients $(7.4 \%$ vs. $5.6 \%)$, as were BSIs caused by fungi $(1.7 \%$ vs. $1.2 \%)$. Altogether, 3,150 (1.8\%) BSIs were caused by 3,168 MDR microbes; 2,503 (1.4\%) BSIs were caused by ESBL-E. coli or ESBL-K. pneumoniae, $562(0.3 \%)$ by MRSA, $66(0.04 \%)$ by VRE, and $37(0.02 \%)$ by CPE. Among 18 BSIs, 2 different MDR microbes were identified.

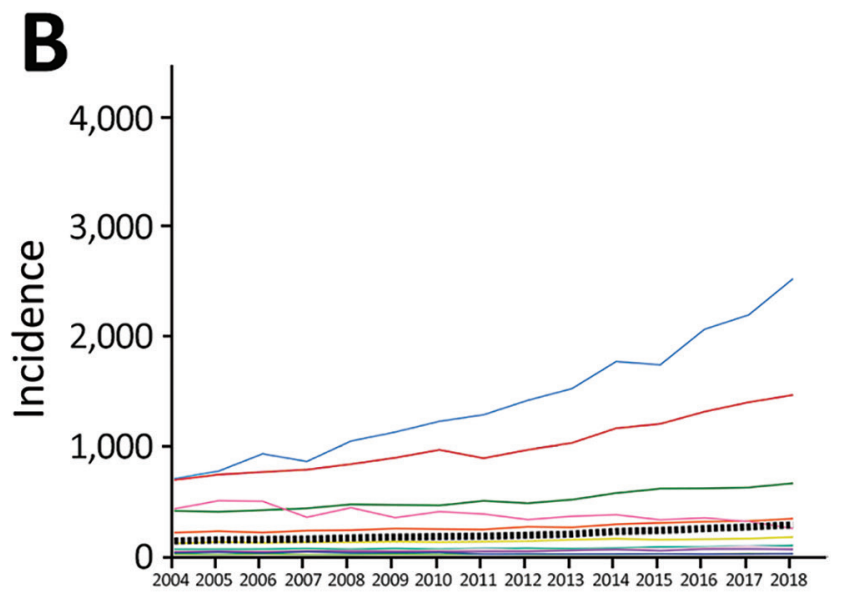

Figure 2. Annual incidence (cases/100,000 population) of bloodstream infections, by sex and age group, Finland, 2004-2018. A) Male patients; B) female patients. 


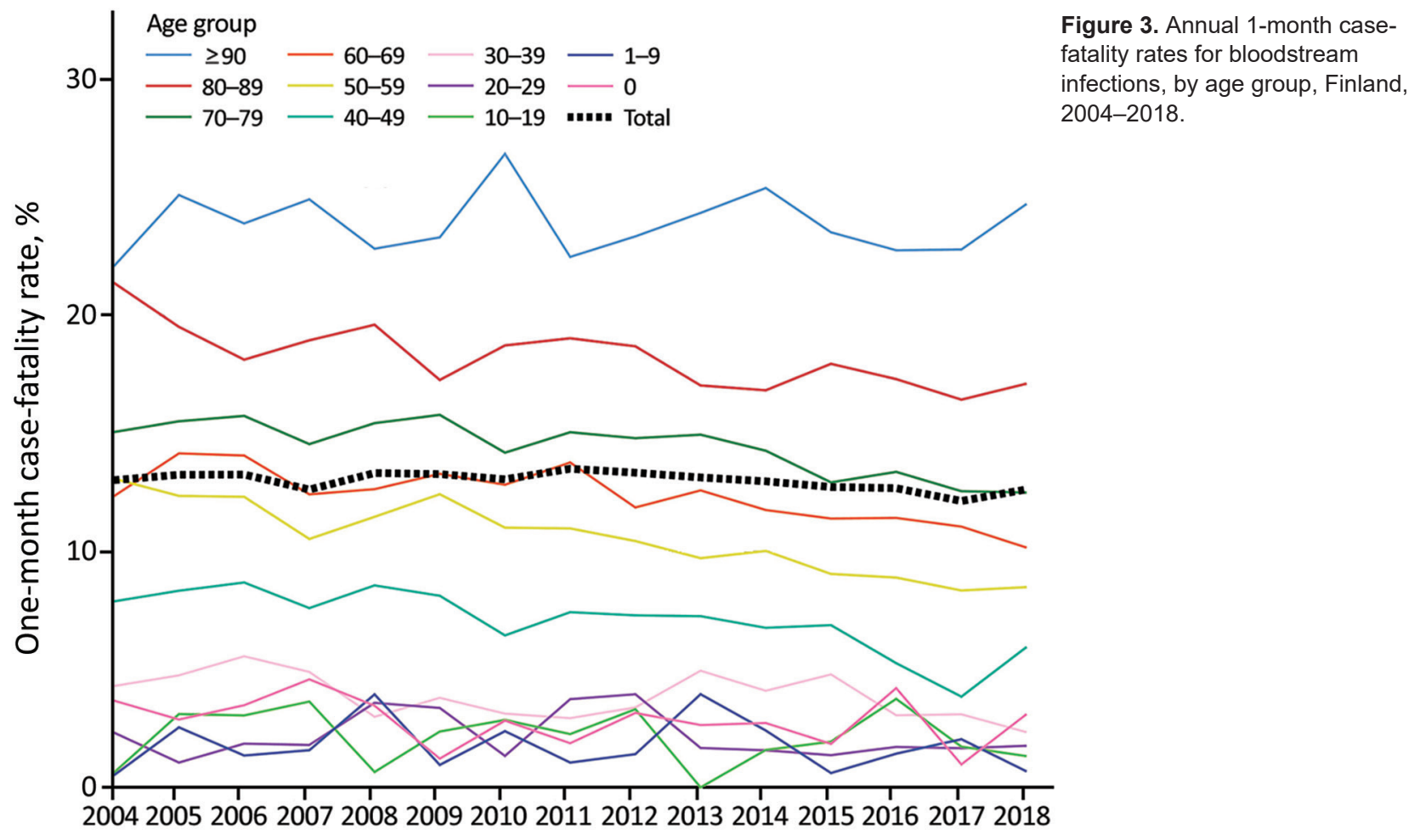

During 2004-2018, the proportion of BSIs caused by gram-negative bacteria rose from $42 \%$ to $48 \%$, whereas BSIs caused by gram-positive bacteria decreased from $50 \%$ to $43 \%$ (Figure 5). Polymicrobial BSIs increased slightly from $6.5 \%$ to $7.1 \%$, and BSIs caused by fungi decreased from $1.7 \%$ to $1.1 \%$ (Figure $5)$. Among the most common pathogens, the proportion of E. coli BSIs rose from $26 \%$ to $30 \%$, whereas no change was noted in S. aureus BSIs (13\%), and we observed a decline in BSIs caused by CNS (from 11\% to $7 \%$ ) and S. pneumoniae (9\% to $4 \%$ ). Candida albicans was the most common fungus, causing $0.9 \%$ of all BSIs and $63 \%$ of fungal BSIs, but the proportion of fungemia caused by other Candida species increased from $34 \%$ to $47 \%$.

The annual incidence of E. coli, S. aureus, $\beta$-hemolytic streptococci, and Klebsiella BSIs rose $>2$-fold. In particular, E. coli BSIs rose from 39 to 91 cases/100,000 population and S. aureus from 19 to 39 cases $/ 100,000$ population. We noted a similar increase in the incidence of E. coli and S. aureus BSIs in both genders and the most prominent increase occurred among persons $>80$ years of age. The incidence of $S$. pneumoniae BSIs rose during 2004-2008, from 13 cases/100,000 population to 17 cases $/ 100,000$ population, and then decreased to 13 cases/100,000 population in 2018. We observed this decline in all age groups, but we noted the steepest reduction among persons $<30$ years of age, including infants $<1$ year of age.

The proportion of BSIs caused by MDR microbes rose from $0.4 \%$ in 2004 to $2.8 \%$ in 2018 , mostly because of the increase in ESBL-E. coli BSIs, from 0 to $7 \%$ of all E. coli BSIs, an increase from 0 to $10 \%$ among male patients and from 0 to $6 \%$ in female patients. On the other hand, MRSA BSIs decreased from 3.1\% to $2.0 \%$ of all S. aureus BSIs, and the annual number of MRSA BSIs ranged from 27 to 49 during 2004-2018. MDR microbes were causative agents in more BSIs leading to death within 30 days compared with other BSIs $(2.4 \%$ vs. $1.7 \%)$.

Of the 173,715 BSI cases, 123,232 (71\%) were community-acquired and 50,483 (29\%) were healthcareassociated. During 2004-2018, the proportion of community-acquired BSIs rose from $67 \%$ to $78 \%$, whereas healthcare-associated BSIs declined from $33 \%$ to $22 \%$. The median CCI of all BSI patients was 1 (range 0-15); $38 \%$ had a low score (CCI 0 ) and $21 \%$ had a high score (CCI $\geq 3$ ). During 2004-2018, the proportion of patients with a high CCI increased from $14 \%$ to $23 \%$, but the proportion of patients with a low CCI decreased from $45 \%$ to $35 \%$.

\section{Discussion}

In our nationwide population-based study, BSI incidence and mortality rates increased 2-fold during 


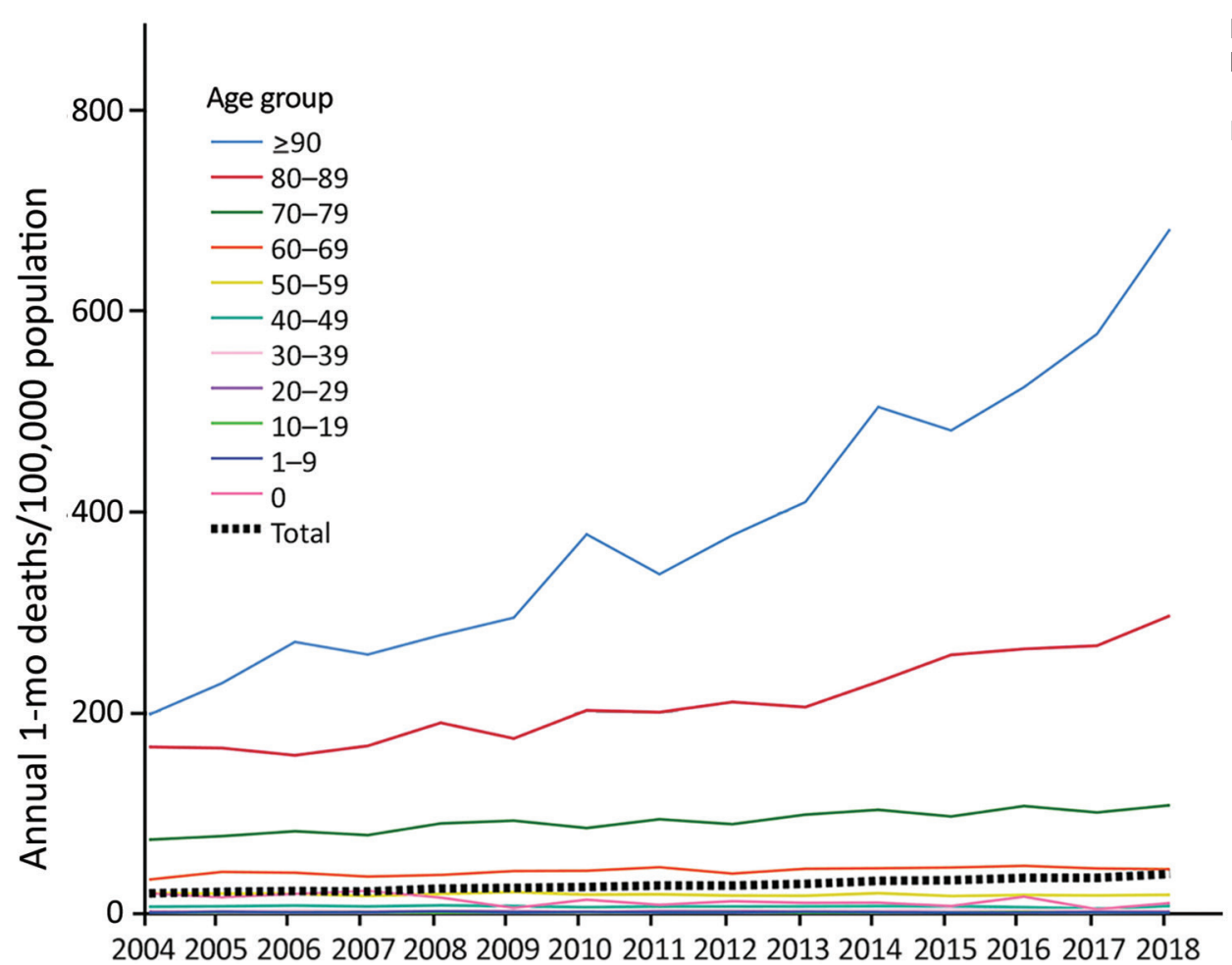

Figure 4. Annual average 1-month bloodstream infection deaths per 100,000 population, by age group, Finland, 2004-2018.

2004-2018 with the sharpest rise among persons $\geq 80$ years of age. Community-acquired BSIs contributed to the rising incidence more than healthcare-associated BSIs did. The 1-month case fatality rate was $13 \%$ and remained rather stable over time despite the growing proportion of patients with high CCI scores. We noted a considerable, $>2$-fold, increase in the incidence of $E$. coli BSIs. The proportion of BSIs caused by MDR microbes was low, but we observed an ascending trend, mainly because of the increase in ESBLE. coli BSIs.

During 2004-2018, the annual incidence of BSIs in Finland rose from 150 to 309 cases/100,000 population with an average annual rate of 216 BSI episodes/100,000 population. In 2 previous nationwide studies from Finland based on the same laboratory surveillance data, the average annual incidence rates were considerably lower than in our study, 125 cases/100,000 population during 1995-2002 and 159 cases/100,000 population during 2004-2007 $(3,20)$. Similar increasing incidence rates have also been noted in other population-based surveys from Europe; from 114 to 166 cases/100,000 person-years during 1992-2006 in northern Denmark (10) and from 190 to 257 cases/100,000 person-years during 2002-2013 in midNorway (6). One recent population-based study, a report from Funen County, Denmark, during 2000-
2008, demonstrated a decreasing overall incidence of BSIs (15).

We observed a marked, nearly 2 -fold increase in all-cause mortality during 2004-2018; however, at the same time, the 1-month case-fatality rate decreased slightly, which might reflect advances in treatment for BSIs. A study from Norway during 2002-2013 demonstrated a similar mortality rate (32 cases/100,000 population) as in our study ( 28 cases $/ 100,000$ population) and showed higher rates in male than in female patients, comparable to our results (6). In that study, case-fatality rates decreased from $17.2 \%$ to $13.1 \%$ between 2002-2007 and 2008-2013 concurrent with an increasing incidence of BSIs and rising rates of blood culture sampling (6). A higher 30-day mortality rate was observed among hospitalized patients with bacteremia in Denmark, but that study also noted decreasing rates from $22.7 \%$ to $20.6 \%$ between $1992-$ 1996 and 2002-2006 (10). A recent study from Sweden during 2000-2013 showed a 1-month case-fatality rate of $12.8 \%$, similar to our results (12).

In our study, the proportion of BSI patients with a low CCI declined during 2004-2018 from $45 \%$ to $35 \%$, but the proportion of those with a high CCI increased from $14 \%$ to $23 \%$. Similarly, in a report from Denmark during 1992-2006, the proportion of BSI patients with a low CCI decreased from $42 \%$ to $33 \%$, and the proportion of those with a high CCI rose from $20 \%$ to 
$30 \%$ (10). Furthermore, in a survey of BSIs from a county in Sweden, the proportion of patients with $\geq 1$ underlying condition increased from $21 \%$ to $55 \%$ during 2000-2013 (12).

The average annual incidence of BSIs in our study was highest among older persons, as demonstrated in previous studies $(1-4,6,21)$. In addition, we noted that the increase in the incidence over time was most notable among the oldest persons, those $\geq 90$ years of age. Researchers widely recognize that the risk for BSIs increases as the population ages and as the life expectancy rises in industrialized countries. It is likely that the considerable rise in BSI incidence over time is also associated with increasing prevalence of underlying conditions, advanced treatments of chronic diseases, and implementation of invasive procedures. We noted a higher average annual BSI incidence among male patients, which aligns with results from previous reports $(1-3,6,21)$, and is presumably related to higher prevalence of chronic diseases and predisposing factors among male persons.

In our study, healthcare-associated BSIs accounted for $29 \%$ of all BSIs and community-acquired
BSIs for $71 \%$; the proportion of healthcare-associated BSIs decreased during 2004-2018, but communityacquired BSIs increased. Similarly, a study in Sweden noted that hospital-acquired BSIs accounted for 33\% and community-onset BSIs for $67 \%$ of all BSIs (12). In a survey from Denmark that reported 3 categories of BSIs by origin, the portion of nosocomial and community-acquired BSIs declined during 1992-2006, but healthcare-associated BSIs increased by $>2$-fold during the same time (10). The 2-day timeframe for our definition of healthcare-associated BSI was rather strict and might have led to underestimation of these BSIs. Some healthcare-associated BSIs possibly were inaccurately interpreted as community-acquired because the data on the origin of the infection were obtained from HILMO. The HILMO hospital discharge registry does not cover long-term care facilities, does not include information on possible outpatient invasive procedures before the BSI, and does not provide data on regular patient hospital visits for chronic hemodialysis or chemotherapy.

E. coli and S. aureus were the most common causative pathogens of all BSIs in our study, and in similar
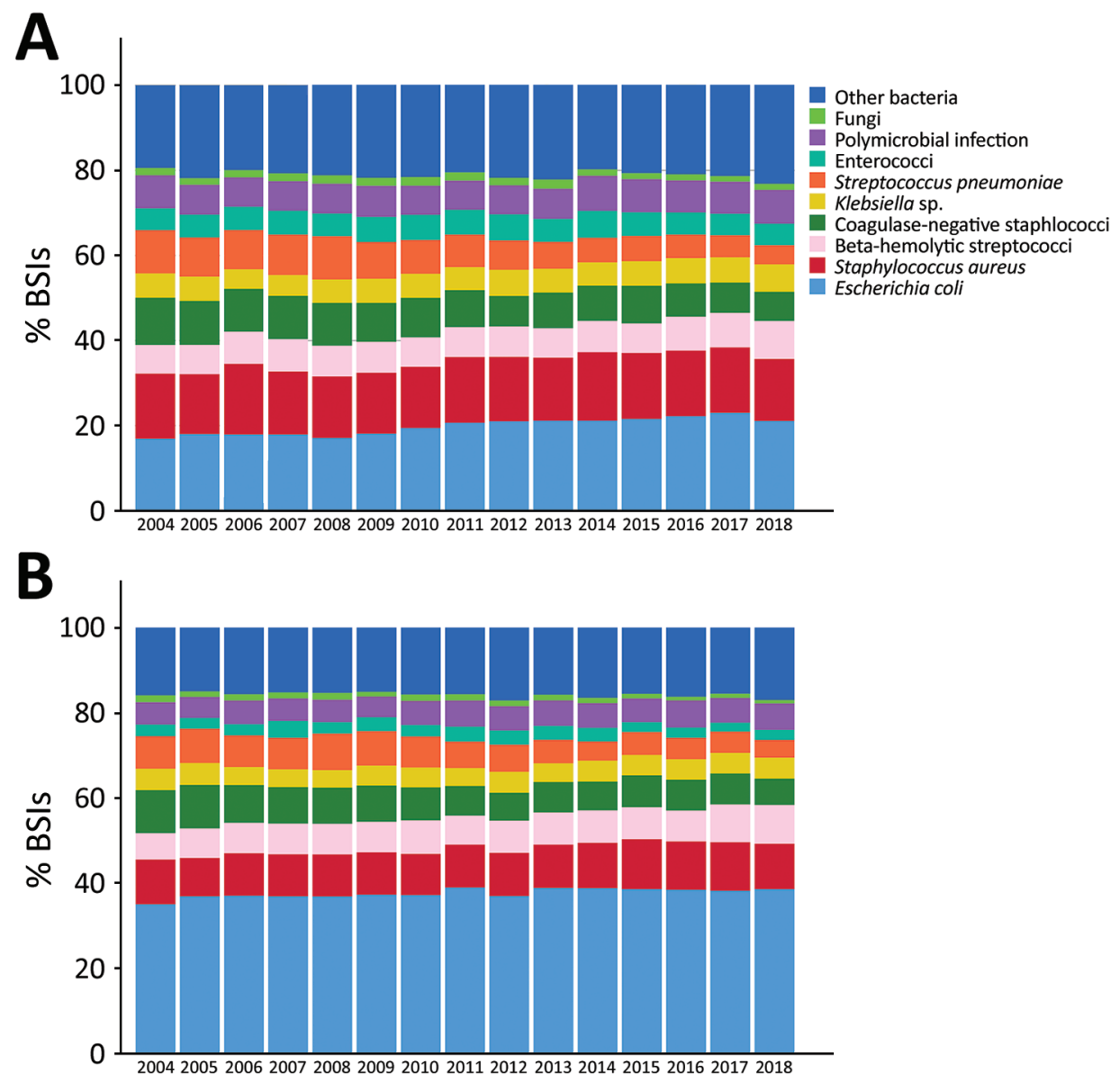

Figure 5. Frequency distribution of the most common causative agents of BSIs, by sex, Finland, 2004-2018. A) Male patients; B) female patients. BSI, bloodstream infections. 
proportions as have been reported from Europe and North America $(1,3,12,15,21)$. Our findings show the proportion of E. coli BSIs increased from $26 \%$ to $30 \%$ during 2004-2018, but no change was noted in S. aureus BSIs (13\%). Similarly, in a report of BSIs in England during 2004-2008, the proportion of E. coli BSIs rose from $19 \%$ to $23 \%$, but $S$. aureus BSIs decreased from $17 \%$ to $12 \%$ because of reduction in methicillin-resistant strains (2). A considerable increase in the proportion of E. coli BSIs also has been observed in Sweden (12), and in 2 other reports from countries in Scandinavia, the prevalence of bacteremia with urinary tract foci increased concurrently with rising rates of $E$. coli BSIs $(6,10)$. In our study, the annual incidence of both E. coli and S. aureus rose by >2-fold during 2004-2018, which is comparable to results from previous population-based surveys $(22,23)$. On the contrary, we noted that the incidence of $S$. pneumoniae BSIs decreased after 2008, which most likely is associated with the implementation of pneumococcal vaccines in Finland (24). This finding is in line with studies from Norway during 2002-2013 and England during 2004-2008 showing reduction in the incidence of S. pneumoniae BSIs after introduction of the conjugate vaccine to the childhood immunization schedule in $2006(2,6)$.

In our study, the proportion of BSIs caused by MDR microbes was low (1.8\%), but we observed a distinct ascending trend during 2004-2018; ESBL-E. coli BSIs increased the most. On the other hand, the proportion of MRSA BSIs decreased over time. In Finland, as in other Nordic countries, the proportion of MDR BSIs is typically low $(6,25,26)$, whereas North America and most of Europe have considerably higher proportions of MDR BSIs, as shown in previous surveillance studies (27-29). These surveys also demonstrate a rising trend in MDR BSIs over time, as noted in our results.

Our study's first limitations was that we did not have exact numbers of blood cultures performed during the study period. However, in a previous report from Finland the annual national blood culturing rate increased by one third during 1995-2002, from 2,752 to 3,667 cultures $/ 100,000$ population (30). Also, estimates from the Finnish Hospital Infection Program suggest that the median number of blood cultures among hospitalized patients in Finland increased by $25 \%$ during $2014-2018$, from $\approx 120$ to 150 blood culture sets/1,000 patient-days (28; O. Lyytikäinen, unpub. data). Recent reports from other countries have shown that increasing blood culture rates might influence the rising BSI incidence $(6,7)$. Thus, higher culturing rates lead to improved detection of milder BSIs, which might contribute to the slightly decreasing case-fatality rates noted in our study. The rising incidence of BSIs also might reflect changes in the healthcare delivery system, such as centralized healthcare services in which patients with acute infections are treated at hospital emergency departments instead of in community healthcare centers, and blood cultures possibly are taken before patient conditions progress to severe BSI or when patients have milder infections and milder symptoms. Second, because the study was based on surveillance data, we did not have information on the focus of the infection, on possible delays in recognition and treatment of the infection, nor data on the appropriateness of antimicrobial therapy, which might have affected the outcome of BSIs. Third, we did not have information on the main cause of death of the patients, but presumably BSI was a contributing factor. Finally, we did not have data on patients' underlying medical conditions other than those included in the CCI, nor did we have information on possible do-not-resuscitate orders for patients, which likely have influenced patient outcome, as we observed in our previous population-based case series of BSIs leading to early death (31).

Our population-based study of $>170,000$ BSIs in Finland during 15 consecutive years offers a comprehensive assessment of temporal trends and outcome of BSIs. We noted a 2-fold rise in the incidence and BSI mortality rates during 2004-2018. The proportion of BSIs caused by resistant microbes, mostly by ESBL-E. coli, rose over time, which could complicate antimicrobial therapy in the future and increase the risk for fatal BSI outcomes. Further research is required to assess the possible predisposing factors for BSI mortality. Overall, issues related to the increasing BSI incidence and death raised in our study ought to be evaluated separately in cases of community-acquired and healthcare-associated BSI in the future. Nonetheless, our data serve as a valuable point of reference for industrialized countries when estimating the effects of changes in the epidemiology of BSIs among an aging population and increasing antimicrobial resistance. Continuous BSI surveillance is needed to compose local recommendations for empiric antimicrobial treatment. Our findings underscore the necessity for substantial BSI prevention efforts and increased public and healthcare system awareness of severe infections.

K.K. received grants from the Finnish Cultural Foundation (no. 00190532), from the Finnish Society for Study of Infectious Diseases (no. 04/09/2019), from the HUS Inflammation Center Research Fund (no. Y1209INF01, Y1209TUTKK), and from state funding for the Finnish University Hospitals (no. TYH2018108). 


\section{About the Author}

Dr. Kontula is a specialist in infectious diseases at the Helsinki University Hospital. Her research interests include the epidemiology and outcome of bloodstream infections.

\section{References}

1. Uslan DZ, Crane SJ, Steckelberg JM, Cockerill FR III, St Sauver JL, Wilson WR, et al. Age- and sex-associated trends in bloodstream infection: a population-based study in Olmsted County, Minnesota. Arch Intern Med. 2007;167:8349. https://doi.org/10.1001/archinte.167.8.834

2. Wilson J, Elgohari S, Livermore DM, Cookson B, Johnson A, Lamagni T, et al. Trends among pathogens reported as causing bacteraemia in England, 2004-2008. Clin Microbiol Infect. 2011;17:451-8. https://doi.org/10.1111/ j.1469-0691.2010.03262.x

3. Skogberg K, Lyytikäinen O, Ollgren J, Nuorti JP, Ruutu P. Population-based burden of bloodstream infections in Finland. Clin Microbiol Infect. 2012;18:E170-6. https://doi.org/10.1111/j.1469-0691.2012.03845.x

4. Laupland KB. Defining the epidemiology of bloodstream infections: the 'gold standard' of population-based assessment. Epidemiol Infect. 2013;141:2149-57. https://doi.org/10.1017/S0950268812002725

5. Buetti N, Atkinson A, Marschall J, Kronenberg A; Swiss Centre for Antibiotic Resistance (ANRESIS). Incidence of bloodstream infections: a nationwide surveillance of acute care hospitals in Switzerland 2008-2014. BMJ Open. 2017;7:e13665. https://doi.org/10.1136/bmjopen-2016-013665

6. Mehl A, Åsvold BO, Lydersen S, Paulsen J, Solligård E, Damås JK, et al. Burden of bloodstream infection in an area of mid-Norway 2002-2013: a prospective population-based observational study. BMC Infect Dis. 2017;17:205. https:/ / doi.org/10.1186/s12879-017-2291-2

7. Laupland KB, Niven DJ, Pasquill K, Parfitt EC, Steele L. Culturing rate and the surveillance of bloodstream infections: a population-based assessment. Clin Microbiol Infect. 2018;24:910. e1-4. https://doi.org/10.1016/j.cmi.2017.12.021

8. Goto M, Al-Hasan MN. Overall burden of bloodstream infection and nosocomial bloodstream infection in North America and Europe. Clin Microbiol Infect. 2013;19:501-9. https://doi.org/10.1111/1469-0691.12195

9. Rodríguez-Baño J, López-Prieto MD, Portillo MM, Retamar P, Natera C, Nuño E, et al.; SAEI/SAMPAC Bacteraemia Group. Epidemiology and clinical features of community-acquired, healthcare-associated and nosocomial bloodstream infections in tertiary-care and community hospitals. Clin Microbiol Infect. 2010;16:1408-13. https://doi.org/10.1111/j.1469-0691.2010.03089.x

10. Søgaard M, Nørgaard M, Dethlefsen C, Schønheyder HC. Temporal changes in the incidence and 30-day mortality associated with bacteremia in hospitalized patients from 1992 through 2006: a population-based cohort study. Clin Infect Dis. 2011;52:61-9. https://doi.org/10.1093/cid/ciq069

11. Lenz R, Leal JR, Church DL, Gregson DB, Ross T, Laupland KB. The distinct category of healthcare associated bloodstream infections. BMC Infect Dis. 2012;12:85. https://doi.org/10.1186/1471-2334-12-85

12. Holmbom M, Giske CG, Fredrikson M, Östholm Balkhed Å, Claesson C, Nilsson LE, et al. 14-year survey in a Swedish county reveals a pronounced increase in bloodstream infections (BSI). Comorbidity - an independent risk factor for both BSI and mortality. PLoS One. 2016;11:e0166527. https:/ / doi.org/10.1371/journal.pone.0166527
13. Laupland KB, Davies HD, Church DL, Louie TJ, Dool JS, Zygun DA, et al. Bloodstream infection-associated sepsis and septic shock in critically ill adults: a population-based study. Infection. 2004;32:59-64. https://doi.org/10.1007/ s15010-004-3064-6

14. Prowle JR, Echeverri JE, Ligabo EV, Sherry N, Taori GC, Crozier TM, et al. Acquired bloodstream infection in the intensive care unit: incidence and attributable mortality. Crit Care. 2011;15:R100. https:/ / doi.org/10.1186/cc10114

15. Nielsen SL, Pedersen C, Jensen TG, Gradel KO, Kolmos HJ, Lassen AT. Decreasing incidence rates of bacteremia: a 9-year population-based study. J Infect. 2014;69:51-9. https://doi.org/10.1016/j.jinf.2014.01.014

16. Laupland KB, Church DL. Population-based epidemiology and microbiology of community-onset bloodstream infections. Clin Microbiol Rev. 2014;27:647-64. https://doi.org/ 10.1128/CMR.00002-14

17. Laupland KB, Lyytikäinen $\mathrm{O}$, Søgaard M, Kennedy KJ, Knudsen JD, Ostergaard C, et al.; International Bacteremia Surveillance Collaborative. The changing epidemiology of Staphylococcus aureus bloodstream infection: a multinational population-based surveillance study. Clin Microbiol Infect. 2013;19:465-71. https:// doi.org/10.1111/ j.1469-0691.2012.03903.x

18. Charlson ME, Pompei P, Ales KL, MacKenzie CR. A new method of classifying prognostic comorbidity in longitudinal studies: development and validation. J Chronic Dis. 1987;40:373-83. https:/ / doi.org/10.1016/ 0021-9681(87)90171-8

19. Quan H, Sundararajan V, Halfon P, Fong A, Burnand B, Luthi JC, et al. Coding algorithms for defining comorbidities in ICD-9-CM and ICD-10 administrative data. Med Care. 2005;43:1130-9. https://doi.org/10.1097/01. mlr.0000182534.19832.83

20. Skogberg K, Lyytikäinen O, Ruutu P, Ollgren J, Nuorti JP. Increase in bloodstream infections in Finland, 1995-2002. Epidemiol Infect. 2008;136:108-14. https://doi.org/10.1017/ S0950268807008138

21. Laupland KB, Pasquill K, Parfitt EC, Naidu P, Steele L. Burden of community-onset bloodstream infections, Western Interior, British Columbia, Canada. Epidemiol Infect. 2016;144:2440-6. https://doi.org/10.1017/S0950268816000613

22. Williamson DA, Lim A, Wiles S, Roberts SA, Freeman JT. Population-based incidence and comparative demographics of community-associated and healthcare-associated Escherichia coli bloodstream infection in Auckland, New Zealand, 2005-2011. BMC Infect Dis. 2013;13:385. https:/ / doi.org/10.1186/1471-2334-13-385

23. Thorlacius-Ussing L, Sandholdt H, Larsen AR, Petersen A, Benfield T. Age-dependent increase in incidence of Staphylococcus aureus bacteremia, Denmark, 2008-2015. Emerg Infect Dis. 2019;25:875-82. https:/ / doi.org/10.3201/ eid2505.181733

24. Rinta-Kokko H, Palmu AA, Auranen K, Nuorti JP, Toropainen M, Siira L, et al. Long-term impact of 10-valent pneumococcal conjugate vaccination on invasive pneumococcal disease among children in Finland. Vaccine. 2018;36:1934-40. https://doi.org/10.1016/ j.vaccine.2018.03.001

25. European Centre for Disease Prevention and Control (ECDC). Surveillance of antimicrobial resistance in Europe 2018. Annual report of the European Antimicrobial Resistance Surveillance Network (EARS-Net). Stockholm: ECDC. Nov 2019 [cited 2020 Nov 10]. https:/ / www.ecdc. europa.eu/sites/default/files/documents/ surveillance-antimicrobial-resistance-Europe-2018.pdf 
26. Holmbom M, Möller V, Nilsson LE, Giske CG, Rashid MU, Fredrikson M, et al. Low incidence of antibiotic-resistant bacteria in south-east Sweden: an epidemiologic study on 9268 cases of bloodstream infection. PLoS One. 2020;15:e0230501. https://doi.org/10.1371/journal.pone.0230501

27. Wisplinghoff $\mathrm{H}$, Bischoff $\mathrm{T}$, Tallent SM, Seifert $\mathrm{H}$, Wenzel RP, Edmond MB. Nosocomial bloodstream infections in US hospitals: analysis of 24,179 cases from a prospective nationwide surveillance study. Clin Infect Dis. 2004;39:309-17. https://doi.org/10.1086/421946

28. de Kraker ME, Jarlier V, Monen JC, Heuer OE, van de Sande N, Grundmann H. The changing epidemiology of bacteraemias in Europe: trends from the European Antimicrobial Resistance Surveillance System. Clin Microbiol Infect. 2013;19:860-8. https://doi.org/10.1111/14690691.12028

29. Diekema DJ, Hsueh PR, Mendes RE, Pfaller MA, Rolston KV, Sader HS, et al. The microbiology of bloodstream infection: 20-year trends from the SENTRY Antimicrobial Surveillance Program. Antimicrob Agents Chemother. 2019;63:e00355-19. https:// doi.org/10.1128/AAC.00355-19

30. Klemets P, Lyytikäinen O, Ruutu P, Kaijalainen T, Leinonen M, Ollgren J, et al. Trends and geographical variation in invasive pneumococcal infections in Finland. Scand J Infect Dis. 2008;40:621-8. https:/ / doi.org/ $10.1080 / 00365540801938931$

31. Kontula KS, Skogberg K, Ollgren J, Järvinen A,

Lyytikäinen O. Early deaths in bloodstream infections: a population-based case series. Infect Dis (Lond). 2016;48:379_ 85. https://doi.org/10.3109/23744235.2015.1131329

Address for correspondence: Keiju Kontula, Division of Infectious Diseases, Inflammation Center, Helsinki University Hospital, P.O. Box 340, 00029 HUS, Helsinki, Finland; email: keiju.kontula@hus.fi

\section{Emerging Infectious Diseases Spotight Topics}
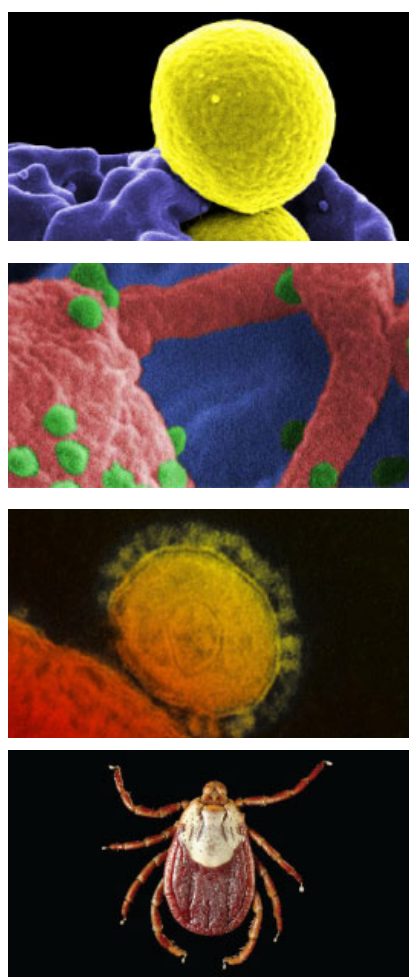
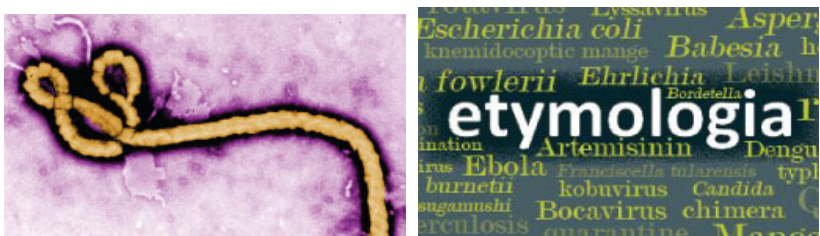

Antimicrobial resistance • Ebola Etymologia • Food safety $\bullet$ HIV-AIDS Influenza • Lyme disease • Malaria MERS • Pneumonia • Rabies • Ticks Tuberculosis • Coronavirus • Zika
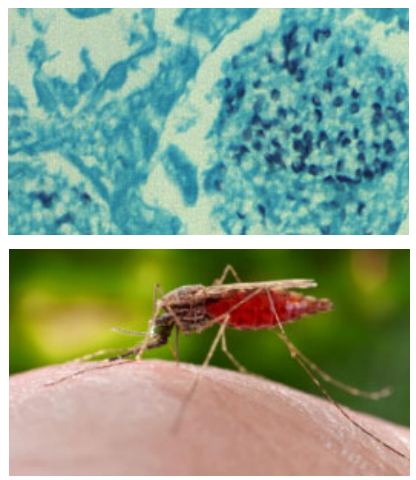
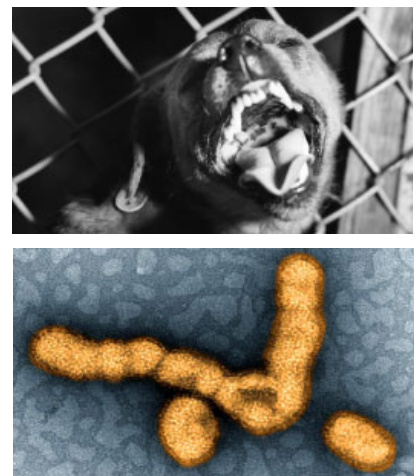
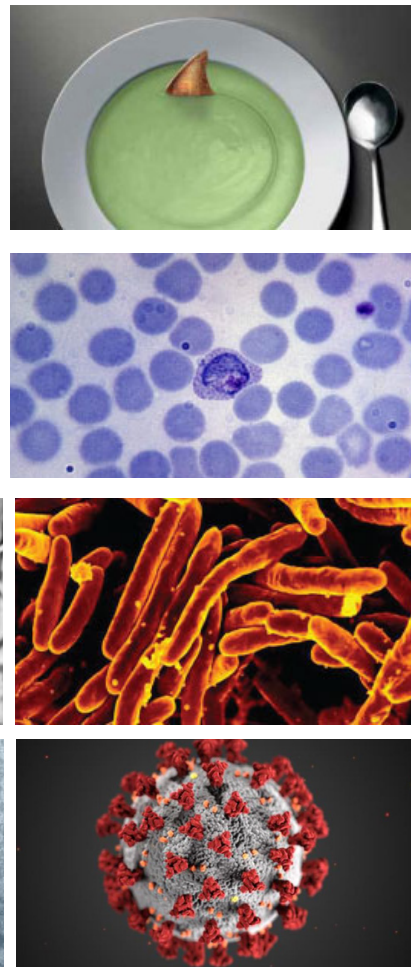

EID's spotlight topics highlight the latest articles and information on emerging infectious disease topics in our global community

https://wwwnc.cdc.gov/eid/page/spotlight-topics 